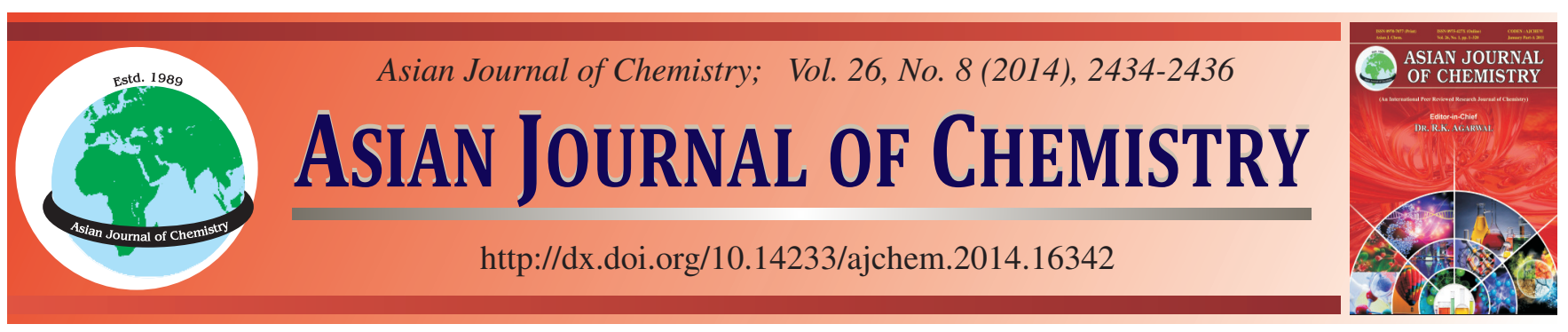

\title{
Simultaneous Determination of Three Active Ingredients in Schisandra chinensis (Turcz.) Baill by HPLC
}

\author{
Weiming $\mathrm{CHENG}^{1}$, Jie GAO ${ }^{2}$ and Shan ZhONG ${ }^{3, *}$
}

\begin{abstract}
${ }^{1}$ Zhejiang Institute for Food and Drug Control, No 86 Lane 1 Jichang Road Jianggan Dist. 310004, Hangzhou Zhejiang Province, P.R. China ${ }^{2}$ Grade 2011, Zhejiang University of TCM, No. 548, Binwen Road, Binjiang Dist. 310053, Hangzhou Zhejiang Province, P.R. China

${ }^{3}$ State Key Laboratory of Respiratory Disease, The First Affiliated Hospital of Guangzhou Medical University (Guangzhou Medical University), No. 151 YanJiang Road Yuexiu Dist. 510120, Guangzhou, Guangdong Province, P.R. China
\end{abstract}

*Corresponding author: Tel: +86 18664535213; E-mail: zhong-shan2000@ hotmail.com

In present study, an HPLC method was setup for the simultaneous determination of 3 active ingredients in Schisandra chinensis (Turcz.) Baill. HPLC conditions included Kromasil- $\mathrm{C}_{18}$ column $(250 \times 4.6 \mathrm{~mm}, 5 \mu \mathrm{m})$ and the mobile phase was a mixture of $\mathrm{MeOH}-\mathrm{CH}_{3} \mathrm{CN}-\mathrm{H}_{2} \mathrm{O}$ (5:1:4). The flow rate was $1 \mathrm{~mL} / \mathrm{min}$, the column temperature was at $30^{\circ} \mathrm{C}$ and the UV detection wavelength was $246 \mathrm{~nm}$. The 3 active ingredients had good linear relationships i.e., schizandrin in the range of 0.10-6.0 $\mu \mathrm{g}\left(\mathrm{R}^{2}=0.9998\right)$, deoxyschizandrin in the range of $0.15-9.0 \mu \mathrm{g}\left(\mathrm{R}^{2}=0.9992\right)$ and schizandrin $\mathrm{B}$ in the range of 0.05-3.0 $\mu \mathrm{g}\left(\mathrm{R}^{2}=0.9997\right)$. Their average recoveries were $99.3 \%(\mathrm{RSD}=1.3$ $\%), 97.1 \%(\mathrm{RSD}=2 \%)$ and $95.2 \%(\mathrm{RSD}=2 \%)$, respectively. This method was steady with high precision and good repeatability and could be used for the determination of the 3 active ingredients and quality control of $S$. chinensis.

Keywords: Schisandra chinensis, Schizandrin, Deoxyschizandrin, Schizandrin B, HPLC.

\section{INTRODUCTION}

Schisandra chinensis (Turcz.) Baill, also known as "Beiwuweizi" in China, belongs to the Schisandraceae family, with the dry mature fruits as the medicament portions. $S$. chinensis mainly distributes in east Asia, southeast Asia and southern parts of north America. It has been used in clinic for a long period in the treatment of insomnia ${ }^{1}$, cough and asthma ${ }^{2}$, hyperglycemia ${ }^{3}$ and hepatitis ${ }^{4}$, etc. Modern pharmacological researches have also confirmed such bioactivities of S. chinensis as immunological enhancement, anti-tumor, cardiovascular enhancement, hypoxia endurance improvement, etc. ${ }^{5-10}$.

As the main medicinal ingredients of $S$. chinensis $^{11}$, lignan exhibits various interesting bioactivities, such as anti-tumor, anti-virus, hepatoprotection and anti-aging. Among the lignin, schizandrin, deoxyschizandrin and schizandrin B are 3 main components, possessing the above bioactivities. In this study, a steady HPLC method, with high precision and good repeatability, was set up for the simultaneous determination of the above 3 active components inside $S$. chinensis, aiming to provide a rapid, simple and precise method for the quality control of S. chinensis.

\section{EXPERIMENTAL}

Agilent 1100 series HPLC system (Angilent Technologies, USA) was equipped with quaternary pump (G1311A), automatic sampler (G1313A), UV variable-wavelength detector (1314A-UV) and column oven (CTO-10ASVP); Pa2251 electronic analytical balance from Sartorius Group, Germany; Tw20 constant temperature bath box from Julabo Labortechnik GmbH Company, Germany.

Standards of schizandrin, deoxyschizandrin and schizandrin B were supplied by National Institute for Food and Drug Control (NIFDC, Beijing, China) with the batch number as 110857-200507, 110764-200408, 110765-200508, respectively. Three batches of $S$. chinensis were purchased from Haozhou Market of Meteria Medica (Batch No: 20100425, 20100827 and 20110911), the voucher specimens were identified by Pharmacogonist Yiguo Sun and kept under certain conditions for future identification. Methanol and acetonitrile were of HPLC grade and other reagents used were of analytical grade. Deionized water was prepared using a Millipore water purification system.

HPLC conditions: The separation of the 3 ingredients was carried out on a Kromasil- $\mathrm{C}_{18}(250 \mathrm{~mm} \times 4.6 \mathrm{~mm}, 5 \mu \mathrm{m})$. 
The solvents used for HPLC separation were MeOH$\mathrm{CH}_{3} \mathrm{CN}-\mathrm{H}_{2} \mathrm{O}(5: 1: 4)$ and the analysis was monitored at 246 $\mathrm{nm}$ with the column temperature of $30{ }^{\circ} \mathrm{C}$ and the injection volume was $20 \mu \mathrm{L}$.

Preparation of standard solution: Certain amounts of the 3 standard compounds were dissolved with methanol into volumetric flasks, respectively. Then measured $1 \mathrm{~mL}$ schizandrin, $1 \mathrm{~mL}$ deoxyschizandrin and $1.5 \mathrm{~mL}$ schizandrin B standard solution, respectively, into a $50 \mathrm{~mL}$ volumetric flask and diluted to the concentrations of 100.3, 150.4 and $51.3 \mu \mathrm{g} / \mathrm{mL}$.

Preparation of sample solution: $10 \mathrm{~g}$ dried S. chinensis was finely ground into powder ( $80 \mathrm{mesh}$ ), about $0.5 \mathrm{~g}$ sample was accurately weighted, then added into a $50 \mathrm{~mL}$ conical flask. $20 \mathrm{~mL} \mathrm{MeOH}$ was added into the flask and performed ultrasonic extraction in a constant temperature bath for $0.5 \mathrm{~h}$. The extract solution was then cooled to room temperature and diluted to the volume. After centrifuged at $12000 \mathrm{rpm}$ for 10 min, the supernatant was passed through a $0.22 \mu \mathrm{m}$ membrane filter and the filtrate was ready for the chromatographic analysis.

\section{RESULTS AND DISCUSSION}

Linear regression analysis for each component was performed by the external standard method. The above 3 compound solution was accurately injected 1, 5, 10, 20, 30 and $60 \mu \mathrm{L}$, respectively. The linearity of each compound was calculated by plotting the peak area (Y) vs. concentration $(\mathrm{X})$. (Table-1). All the 3 components showed good linearities in wide concentration ranges.

\section{TABLE-1}

\section{LINEAR REGRESSION EQUATION AND LINEAR RANGES}

\begin{tabular}{cccc}
\hline Components & $\begin{array}{c}\text { Regression } \\
\text { equation }\end{array}$ & $\mathrm{R}^{2}$ & $\begin{array}{c}\text { Linear range } \\
(\mu \mathrm{g})\end{array}$ \\
\hline Schizandrin & $\mathrm{Y}=11.22 \mathrm{X}+1.76$ & 0.9998 & $0.10 \sim 6.0$ \\
Deoxyschizandrin & $\mathrm{Y}=8.31 \mathrm{X}+2.55$ & 0.9992 & $0.15 \sim 9.0$ \\
Schizandrin B & $\mathrm{Y}=7.09 \mathrm{X}+2.11$ & 0.9997 & $0.05 \sim 3.0$ \\
\hline
\end{tabular}

Precision: The standard mixture solution of the 3 components was injected into HPLC six times continuously and the area of each peak was used for the calculation of precision (Fig. 1 ). The results showed that relative stand deviation (RSD) of peak area of each standard was $1.3,1.4$ and $2.1 \%$, respectively.

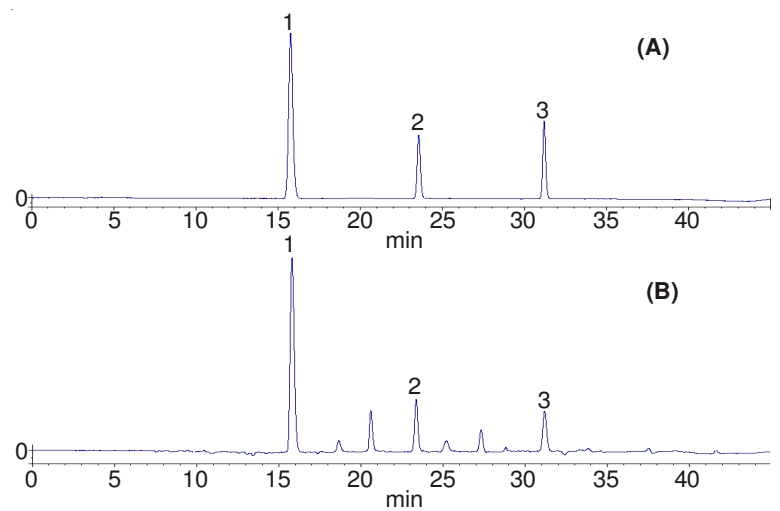

Fig. 1. Typical HPLC chromatograms of the 3 ingredients mixture (a) and $S$. chinensis (b) 1: schizandrin; 2: deoxyschizandrin; 3: schizandrin B

Stability: As for the stability test, the same sample solution was analyzed at designated time points in $24 \mathrm{~h}$. The results showed that RSDs of peak areas were $0.9,1.3$ and $1.5 \%$ and found to be stable for the experiment.

Repeatability: Repeatability was carried out using six samples solution after the same treatment procedure. The results showed that RSD of each peak area was 1.1, 0.8 and $1.2 \%$, respectively.

Recovery test: The sample with known targeted contents was spiked with certain amounts of the 3 standards. Then the spiked sample was processed in accordance with the established method for the HPLC detection. The average recoveries for schizandrin, deoxyschizandrin and schizandrin B determined were 95.2-99.3\% (Table-2).

Application of the HPLC method for the quantitation studies: The experiment determined 3 samples of different batches with the above method. The contents of the 3 components were shown in Table-3.

\begin{tabular}{|c|c|c|c|c|c|c|}
\hline \multicolumn{7}{|c|}{$\begin{array}{c}\text { TABLE }-2 \\
\text { RECOVERY RESULTS OF THE } 3 \text { ACTIVE INGREDIENTS }\end{array}$} \\
\hline Component & Contents in samples (mg) & Added (mg) & Found (mg) & Recovery (\%) & Mean (\%) & $\operatorname{RSD}(\%)$ \\
\hline \multirow{6}{*}{ Schizandrin } & 0.7156 & 0.6933 & 1.4034 & 99.2 & \multirow{6}{*}{99.3} & \multirow{6}{*}{1.3} \\
\hline & 0.7033 & 0.6933 & 1.3807 & 97.7 & & \\
\hline & 0.6905 & 0.6933 & 1.3762 & 98.9 & & \\
\hline & 0.7191 & 0.6933 & 1.4117 & 99.9 & & \\
\hline & 0.6832 & 0.6933 & 1.3869 & 101.5 & & \\
\hline & 0.7200 & 0.6933 & 1.4036 & 98.6 & & \\
\hline \multirow{6}{*}{ Deoxyschizandrin } & 0.1112 & 0.1034 & 0.2106 & 96.1 & \multirow{6}{*}{97.1} & \multirow{6}{*}{2.0} \\
\hline & 0.1093 & 0.1034 & 0.2075 & 95.0 & & \\
\hline & 0.1073 & 0.1034 & 0.2089 & 98.3 & & \\
\hline & 0.1117 & 0.1034 & 0.2141 & 99.0 & & \\
\hline & 0.1062 & 0.1034 & 0.2087 & 99.2 & & \\
\hline & 0.1119 & 0.1034 & 0.2101 & 95.0 & & \\
\hline \multirow{6}{*}{ Schizandrin B } & 0.0940 & 0.0858 & 0.1765 & 96.1 & \multirow{6}{*}{95.2} & \multirow{6}{*}{2.0} \\
\hline & 0.0924 & 0.0858 & 0.1737 & 94.8 & & \\
\hline & 0.0907 & 0.0858 & 0.1742 & 97.3 & & \\
\hline & 0.0945 & 0.0858 & 0.1773 & 96.6 & & \\
\hline & 0.0897 & 0.0858 & 0.1688 & 92.1 & & \\
\hline & 0.0946 & 0.0858 & 0.1755 & 94.3 & & \\
\hline
\end{tabular}




\begin{tabular}{cccc}
\hline \multicolumn{5}{c}{ TABLE-3 } \\
CONTENTS DETERMINATION OF THE 3 ACTIVE \\
INGREDIENTS IN 3 BATCHES \\
\hline Batch & $\begin{array}{c}\text { Schizandrin } \\
(\mathrm{mg} / \mathrm{g})\end{array}$ & $\begin{array}{c}\text { Deoxyschizandrin } \\
(\mathrm{mg} / \mathrm{g})\end{array}$ & $\begin{array}{c}\text { Schizandrin B } \\
(\mathrm{mg} / \mathrm{g})\end{array}$ \\
\hline 20100425 & 0.92 & 0.33 & 0.16 \\
20100827 & 1.17 & 0.20 & 0.11 \\
20110911 & 1.34 & 0.21 & 0.17 \\
\hline
\end{tabular}

Optimization of HPLC separation conditions: In order to get a separation with better resolution of targeted components with shorter analytical time, we compared with three different column temperature: 20,30 and $40{ }^{\circ} \mathrm{C}$, then we found that the higher temperature, the shorter retention time with almost the same resolution, so we chosen $30{ }^{\circ} \mathrm{C}$ finally for protecting the lifespan of the column.

Six chromatographic columns of the same specification were compared in the experiment i.e., Zorbax SB- $\mathrm{C}_{18}$, Kromasil$\mathrm{C}_{18}$, Eclipse- $\mathrm{C}_{18}$, Symmetry Shield $\mathrm{RP}_{18}$, Hypersil- $\mathrm{C}_{18}$ and Extend- $\mathrm{C}_{18}$, according to the effect of separation, Kromasil$\mathrm{C}_{18}(250 \mathrm{~mm} \times 4.6 \mathrm{~mm}, 5 \mu \mathrm{m})$ was used for the further research of the methodology.

Besides, reflux extraction and ultrasonic extraction were investigated in the experiments. Considering the advantages of high efficiency, ultrasonic extraction was chosen as the way to prepare the sample solutions. In the meantime, we compared the efficiency of different elution solvents, namely methanol and chloroform, the results indicated that the 2 solvents almost had the same efficiency. Considering the toxicity and safety, methanol was finally selected as the extract solvent.

\section{Conclusion}

From the content determination of 3 batches $S$. chinensis, it could be found that the batch harvested in August and
September had higher contents of the 3 target compounds, which was consistent with the literature and the conventional harvest experience, indicating that better bioactivities might be obtained. In traditional medical use, only the dried fruits of S. chinensis was applied into the clinic. Our current research revealed that the certain lignans also existed in the leaves and stems and the further research was being carried out for the difference comparison among the fruits, leaves and stems.

In short, 3 active components were quantitatively determined in S. chinensis. On the whole, this developed method was simple, accurate for the determination of schizandrin, deoxyschizandrin and schizandrin B and reliable for the quality control and further efficacy study of $S$. chinensis in clinic.

\section{REFERENCES}

1. W. Lin, Z.X. Huang, G.M. Chen, C.F. Lin and X.C. Zhong, Strait J. Preventive Med., 4, 51 (2009).

2. X.G. Li, Q. Gao, W. Wen, P.F. Zhang, F. Xiao and H.M. Luo, J. Chin. Med. Mater, 28, 156 (2005).

3. C.L. Li, S. Zhang and Z.Q. Liu, J. Zhangjiakou Med. College, 12, 123 (2002).

4. L.H. Yu and G.T. Liu, Prog. Chem., 21, 66 (2009).

5. J. Zaugg, S.N. Ebrahimi, M. Smiesko, I. Baburin, S. Hering and M. Hamburger, Phytochemistry, 72, 2385 (2011).

6. T. Lu, H. Chen, Z. Huang, B. Cai, J. Hu, C. Mao and X. Gong, Pharmacogn. Maganize, 9, 155 (2013).

7. X. Ji, Y. Naito, G. Hirokawa, H. Weng, Y. Hiura, R. Takahashi and N. Iwai, Hypertens. Res., 35, 173 (2012).

8. C.H. Ma, L. Yang, F.J. Yang, W.J. Wang, C.J. Zhao and Y.G. Zu, Int. J. Mol. Sci., 13, 14294 (2012).

9. J. Li, H.- Zhao, X.- Ruan, Y.- Xu, W.- Meng, K.- Li and J.- Zhang, J. Zhejiang Univ. Sci. B, 6, 1210 (2005).

10. E.H. Choi, N. Lee, H.J. Kim, M.K. Kim, S.-G. Chi, D.Y. Kwon and H.S. Chun, Genes. Nutr., 2, 337 (2008).

11. H.J. Yang and S.M. Yang, J. Chin. Med. Mater, 26, 618 (2004). 